\title{
Turbulence Theory for the Dissipative Trapped Electron Instability
}
K. T. Tsang
J. D. Callen
G. Vahala 


\section{DISCLAIMER}

This report was prepared as an account of work sponsored by an agency of the United States Government. Neither the United States Government nor any agency Thereof, nor any of their employees, makes any warranty, express or implied, or assumes any legal liability or responsibility for the accuracy, completeness, or usefulness of any information, apparatus, product, or process disclosed, or represents that its use would not infringe privately owned rights. Reference herein to any specific commercial product, process, or service by trade name, trademark, manufacturer, or otherwise does not necessarily constitute or imply its endorsement, recommendation, or favoring by the United States Government or any agency thereof. The views and opinions of authors expressed herein do not necessarily state or reflect those of the United States Government or any agency thereof. 


\section{DISCLAIMER}

Portions of this document may be illegible in electronic image products. Images are produced from the best available original document. 


\section{Printed in the United States of America. Available from National Technical Information Service \\ U.S. Department of Commerce \\ 5285 Port Royal Road, Springfield, Virginia 22161 \\ Price: Printed Copy $\$ 4.00 ;$ Microfiche $\$ 3.00$}

This report was prepared as an account of work sponsored by the United States Government. Neither the United States nor the Energy Research and Development Administration/United States Nuclear Regulatory Commission, nor any of their employees, nor any of their contractors, subcontractors, or their employees, makes any warranty, express or implied, or assumes any legal liability or responsibility for the accuracy, completeness or usefulness of any information, apparatus, product or process disclosed, or represents that its use would not infringe privately uwiled riylils. 
ORNL/TM-5942

Contract No. W-7405-eng-26

FUSION ENERGY DIVISION

TURBULENCE THEORY FOR THE DISSIPATIVE TRAPPED

ELECTRON INSTABILITY

K. T. Tsang

J. D. Callen

This report was prepotice

This report was prepared as an account of work the United States nor the United States Energo
the Research and Development Administration, nor any of Reser eny ar any warranty, express or implied, or assumes ony legal liability or responsibility for the accuracy, completeneso or usefulness of any information, apparatus, product or process disclosed, or represents that its use would not infringe privately owned rights.

G. Vahala

College of William and Mary, Williamsburg, Virginia

(To be submitted to the Physics of Fluids)

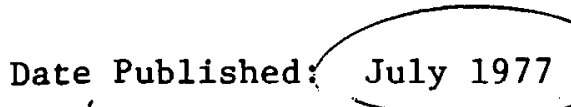

NOTICE This document contains information of a preliminary nature.

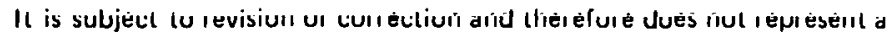
final report.

\author{
Prepared by the \\ OAK RIDGE NATIONAL LABORATORY \\ Oak Ridge, Tennessee \\ operated by \\ UNION CARBIDE CORPORATION \\ for the
}

ENERGY RESEARCH AND DEVELOPMENT ADMINISTRATION 


\section{THIS PAGE}

\section{WAS INTENTIONALLY \\ LEFT BLANK}




\section{CONTENTS}

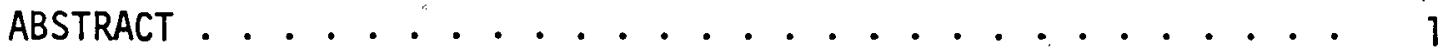

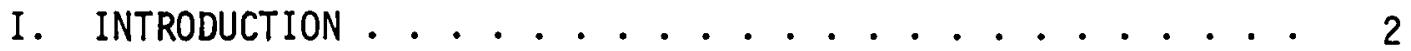

II. TURBULENT TRAPPED ELECTRON RESPONSE. . . . . . . . 6

II. NONLINEAR DISPERSION RELATION. . . . . . . . . . . 14

IV. PARTICLE AND ENERGY FLUXES ................. 16

V. NUMERICAL EXAMPLE AND DISCUSSION ........... 18

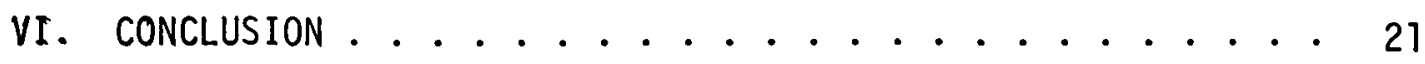

ACKNOWLEDGMENTS. . . . . . . . . . . . . . . 21

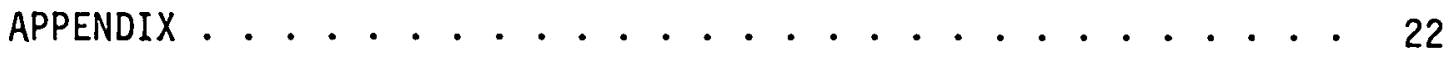

REFERENCES .................... 25 


\begin{abstract}
Drift orbit diffusion induced by turbulence acting on trapped electrons is shown to reduce and broaden the magnetic drift resonance and produce the dominant nonlinear saturation mechanism for the dissipative trapped electron instability. The fluctuation level obtained from such a theory is found to be consistent with present experimental observations.
\end{abstract}




\section{INTRODUCTION}

The existence of drift waves in tokamak-type toroidal confinement devices has been demonstrated by microwave scattering experiments in the Adiabatic Toroidal Compressor ${ }^{1}$ and Tokamak Fontenay-aux-Roses ${ }^{2}$ devices. Because the trapped electron instability is a type of drift wave which is destabilized by trapped electrons, it is expected that it will be operative in larger and hotter tokamaks and will persist even in a reactor regime. The anomalous transport due to this instability is generally believed to be a dominant factor affecting the confinement time of a high temperature plasma. While there is already a fairly complete picture (except for the two-dimensional mode structure) for the linear theory, the nonlinear theory is still rather primitive. The most serious attempt so far has been a one-dimensional, resonant mode-coupling process, ${ }^{3}$ which has difficulty in treating the short wavelength part of the spectrum because of the strong dispersion of the real part of the frequenr.y. In addition, this theory givcs a saturation level which is too high to be consistent with the experimental value. Other nonlinear saturation mechanisms, such as quasi-linear plateauing of the density or temperature profiles, have been observed in numerical simulations. ${ }^{4}$ However, this is highiy improbabie in real experiments because in a tokamak, energy is continuously pumped into the system through ohmic heating or neutral beam injection.

The nonlinear model we investigate in this paper is based on the renormalized turbulence theory of Dupree. ${ }^{5}$ This model is chosen because it apparently gave satisfactory agreement between theory and drift wave experiments in Q-machines. ${ }^{6}$ The major difference between $\mathrm{drift}$ waves in 
Q-machines and those in tokamaks lies in the complexity of the geometry involved. Hence, generalizing Dupree's theory ${ }^{5}$ from slab to tokamak geometry should provide an appropriate nonlinear description for drift waves in a tokamak.

In the original theory on trapped electron modes, ${ }^{7}$ the instability arises purely due to electron collisions. Energy is exchanged between the electrons and the waves through the stochastic process of trapping and detrapping of electrons by pitch angle scattering. Linear stability is attained if the rate of energy transfer from the electrons to the wave is balanced by the rate of radial convection of the wave energy from the rational surface to regions where the waves are ion Landau damped. In this theory, the instability does not exist if there are no collisions.

The picture is significantly changed if the magnetic drift resonance is included in the theory. It is now known that with the inclusion of magnetic drift resonance, the instability persists even without electron collisions. 8 In other words, the trapped electron instability exists in very collisionless plasmas such as the plasma in a reactor regime. The energy exchange between the wave and the electrons is no longer through the random trapping and detrapping of the electrons, but through a Landau type resonance between the trapped electrons and the wave. In the single particle picture, trapped electrons have banana orbits bouncing in poloidal and toroidal directions. At the same time, their average position (or the center of the banana) drifts along the toroidal direction due to the magnetic curvature and gradient. This drift velocity is proportional to the energy of the electron and is in the same direction as the phase velocity of the wave. Therefore, there is always a small group of trapped 
electrons having the correct energy and experiencing a dc electric field on the average. These trapped electrons can exchange energy with the wave just as occurs in the wave-particle resonance in the we11-known theory of Landau resonance. The collisions no longer serve as the catalyst of energy exchange, but rather serve to reduce the strength and broaden the resonance. In fact, as the effective collision frequency of the trapped electrons increases to the same order of magnitude as the trapped electron bounce frequency, the trapped electron destabilizing term in most cases is reduced to a level that can be stabilized by shear in present experimental conditions. 9 In other words, present experiments are not far from the stability boundary of trapped electron instability.

Because collisions play such an important role in reducing the trapped electron destabllizing effect, it is natural to expect in a nonlinear theory that the effective electron collisions caused by the electrostatic turbulence are the dominant mechanism of saturation. This is the nonlinear effect we investigate in this paper. The turbulence effects on the ions are ignored for the time being because the orbit diffusion effect on the ions will change the ion response in such a way that the radial eigenfunction equation for the mode is modified. Such a modification will increase the effective shear and ion viscous damping. If the effective trapped electron coulomb collision frequancy is much smaller than the average trapped electron bounce frequency, the trapped electron destabilizing term in the dispersion relation is much larger than the shear and ion collision terms. In this case, the effect of reducing the trapped electron destabilizing term is more important than enhancing the stabilizing shear and ion colltsion terms. 
In Dupree's turbulence theory of drift waves, a turbulent collision frequency $k_{\perp}^{2} D$ was found, where $k_{\perp}$ is the perpendicular wave number and $D$ is the diffusion coefficient. The meaning of $k_{1}$ is unambiguous in a homogeneous plasma. In an inhomogeneous plasma with gradients perpendicular to the magnetic field, it is not easy to decide which $k_{\perp}$ we should use. In particular, in a tokamak with gradients in the radial direction, it is not clear what combination of $k_{r}$ and $k_{0}$ should appear in $k_{1}^{2} D$. The problem is more complicated if we realize that the eigenmode in the radial direction is not a Fourier sertes but is a sum of parabolic cylinder functions. The radial wave number can only be defined in an average sense. In this paper, we systematically deduce that the turbulent collision is $k_{r}^{2} D$ and find an averaging procedure to obtain this $k_{r}$.

In Sec. II, we derive the turbulent response of the trapped electrons from a bounce averaged drift kinetic equation. The turbulence affects the trapped electron nonadiabatic response mainly through the turbulent collisions. In Sec. III, the nonlinear dispersion relation is derived and the turbulent response of the trapped electrons is treated perturbatively. The calculations show that the inverse of the turning point distance $\left(\mu^{1 / 2}\right)$ is the effective radial wave number that is responsible for the turbulent collisions $k_{r}^{2} D$. Expressions for the particle and energy fluxes due to the instability are given in Sec. IV. Numerical examples are compared with experiments and discussions of the validity of the model are given in Sec. $V$. In the appendix, a general discussion of the distinction between strong (or fluid type) and weak turbulence is also presented. 


\section{TURBULENT TRAPPED ELECTRON RESPONSE}

We use $r, \theta$; and $\zeta$ to denote the radial, poloidal, and toroidal variables. Concentric circular flux surfaces are assumed so that $(r=$ constant) is the equation for a flux surface. Because $\theta$ and $\zeta$ are periodic variables, we can expand any perturbed quantity in a Fourier series in $\theta$ and $\zeta$. The equilibrium unperturbed system has density and temperature gradients in the radial direction. We can decompose any well-behaved radial dependence into a Fourier integral. Thus, the perturbed distribution function $\tilde{f}$ and electrostatic fluctuating potential $\tilde{\phi}$ can be written as

$$
\begin{aligned}
{[\tilde{f}, \tilde{\phi}]=} & \sum_{1, m} \int_{-\infty}^{+\infty} d K\left[f_{1 m}(K), \phi_{1 m}(K)\right] \\
& \exp (i \omega t+i m \theta-i 1 \zeta+i k x),
\end{aligned}
$$

where 1 and $m$ are toroidal and poloidal mode numbers, $K$ is the radial wave number, $x=r-r_{7 m}$, and $r_{7 m}$ is the radial location of the flux surface with safety factor $q\left(r_{7 m}\right)=m / 1$.

In the linear theory, the radial structure of the mode is determined by the shear and ion gyroradius effect. The radial dependence of the trapped electron contribution to the dispersion relation is either ignored or. treated by a perturbation technique. We adopt the same approximation here and assume that in a nonlinear theory, the radial structure is still determined by the ion response." Even including nonlinear effects on ions, the radial eigenmode equation has a parabolic cylinder function as its solution. The derivation is quite lengthy and will be the subject of another paper. The most unstable mode has the form 


$$
\tilde{\phi} \sim \exp \left(i \mu x^{2} / 2\right)
$$

whitch is the same form as in the linear theory. For simplicity and the reasons mentioned in Sec. I, we assume that the ions do not behave very differently from those in the linear theory and write $\mu$ as ${ }^{9}$

$$
\mu=\left|\frac{\dot{k}_{\theta}}{L_{S}}\right| \frac{v_{i}}{\omega \rho_{i}}\left(\frac{S_{1}}{2 S_{2}}\right)^{1 / 2},
$$

where

$$
\begin{aligned}
& S_{1}=\left(\omega-\omega_{*}\right) \Gamma_{0} \omega_{*} n_{i}\left[\Gamma_{0}+b\left(\Gamma_{i}-\Gamma_{0}\right)\right], \\
& \left.S_{2}=\left(\omega-\omega_{*}\right)\left(\Gamma_{0}-\Gamma_{i}\right)-\omega_{*} n_{i}\left[\Gamma_{i}-\Gamma_{0}\right)\right], \\
& \omega_{*}=-\frac{m}{r} \frac{C T}{e B} \frac{\partial \ln N}{\partial r}, \quad L_{S}^{-1}=r(d q / d r) / R q^{2}, \\
& \eta_{i}=d \ln T_{i} / d \ln N, \quad \Omega_{i} \rho_{i}, \\
& \Gamma_{j}=\exp (-b) I_{j}(b), \\
& v_{i}=\left(2 T_{f} / M_{i}\right) 1 / 2, \\
& \rho_{i}=\left(T_{i} / M_{i}\right) 1 / 2 / \Omega_{i}, \\
& k_{\theta}=m / r, \\
& M_{i} \text { is the mass of ions, } T_{i} \text { is the ion temperature, } \\
& I_{i} \text { is the modified Bessel function of order } i, \text { and } \\
& B \text { is the magnitude of the magnetic field. }
\end{aligned}
$$

Then for given 1 and $m$, the radial wave number spectrum is known:

$$
\phi_{1 m}(k)=(2 \pi i \mu)^{-1 / 2} \phi_{1 m} \exp \left(i k^{2} / 2 \mu\right) .
$$

For the trapped electrons, we can write the bounce averaged drift kinetic equation as 


$$
\frac{\partial f}{\partial t}+\frac{c}{r B}\left(\frac{\partial \tilde{\phi}}{\partial r} \frac{\partial}{\partial \theta}-\frac{\partial \tilde{\phi}}{\partial \theta} \frac{\partial}{\partial r}\right) f-\frac{v_{D}}{r} \frac{\partial}{\partial \zeta}\left(f-\frac{e \tilde{\phi}}{T} \bar{f}\right)=c(f) \ldots,
$$

where $f$ and $\bar{f}$ are the total and ensemble averaged trapped electron distribution functions, respectively, and

$$
v_{D}=\frac{v^{2} q H}{2 \Omega_{e} r}
$$

is the drift velocity of the trapped electron in the $\zeta$ direction. The function $\mathrm{H}$ in Eq. (6) is the $G(K)$ defined by Kadomtsev and Pogutse $\mathrm{I}^{\mathrm{U}}$ and depends on both the shear and the pitch angle variable of the trapped electron. When $d$ in $q / d$ In $r \sim 1, H$ is roughly a constant very close to unity. So we are satisfied with taking $v_{D} \approx v^{2} q /\left(2 \Omega_{e} r\right)$. In Eq. (5), we have kept only the important terms of the problem. The second term on the left side is the $E \times B$ drift, and the third term is the drift of the trapped electrons due to magnetic curvature and gradient. A similar equation has been derived by Wadde11.11

If we write $f=\bar{f}+\ddot{f}$ and $\ddot{f}=\sum_{k} f_{k}$, where $k$ denotes the wave vector with fixed $1, m$, and $k$ and $\sum_{k}$ denotes the sum over $1, m$, and the integral over $k$, then the equation for $f_{k}$ is

$$
\frac{\partial f_{k}}{\partial t}+\frac{c}{r B}\left(\frac{\partial \phi_{k}}{\partial r} \frac{\partial}{\partial \theta}-\frac{\partial \phi_{k}}{\partial \theta} \frac{\partial}{\partial r}\right) \bar{f}-\frac{v_{D}}{r} \frac{\partial}{\partial \tau}\left(f_{k}-\frac{e \phi_{k}}{T} \bar{f}\right)=c\left(f_{k}\right)
$$

Next, we define the nonadiabatic response for the trapped electrons $g_{k}$. and use a Krook collision model in such a way that collisions relax the perturbed trapped electron distribution to the adiabatic response,

$$
g_{k} \equiv f_{k}-\frac{e}{T} \phi_{k} \bar{f},
$$




$$
\left(f_{k}\right)=-v\left(f_{k}-\frac{e}{T} \phi_{k} \bar{f}\right),
$$

where $\nu$ is the effective collision frequency for trapped electrons. Then we obtain for $g_{k}$

$$
\begin{aligned}
\left(\frac{\partial}{\partial T}-\frac{v_{D}}{r} \frac{\partial}{\partial \zeta}\right) g_{k}= & \frac{c}{r B}\left(\frac{\partial \phi_{k}}{\partial \theta} \frac{\partial}{\partial r}-\frac{\partial \theta_{k}}{\partial r} \frac{\partial}{\partial \theta}\right) \bar{f} \\
& -\nu g_{k}-\frac{e}{T} \frac{\partial}{\partial t}\left(\phi_{k} \bar{f}\right) .
\end{aligned}
$$

We assume the ensemble average distribution function $\bar{f}$ to be a local Maxwellian

$$
\bar{f}=N(r)\left[m_{e} / 2 \pi T(r)\right]^{3 / 2} \exp \left(-m_{e} v^{2} / 2 T\right) .
$$

Equation (7) can then be written as

$$
\left(\frac{\partial}{\partial T}-\frac{v_{D}}{r} \cdot \frac{\partial}{\partial \zeta}\right) g_{k} \exp (\nu t)=i\left(\omega-\omega_{*}^{T}\right) \frac{e \phi_{k}}{T} \bar{f} \exp (v t),
$$

where $\omega_{*}^{\top}=(-m / r)(C T / e B)(\partial \ln \bar{f} / \partial r)$. In linear theory, Eq. (8) can be solved by integrating the right side along unperturbed orbits of the average position of the trapped electrons (banana center). It is easy to show that Eq. (8) gives the usual linear nonadiabatic trapped electron response. Using the renormalized turbulence theory of Dupree, 5 the nonlinear trapped electron response is obtained by direct analogy with the results in Ref. 5 ,

$$
\begin{aligned}
g_{1 m}(K)= & i\left(\omega-\omega_{*}^{\top}\right) \frac{e \phi_{1 m}(K)}{T} \bar{f} \int_{0}^{m} d \tau \exp [(i \omega-\nu) \tau \\
& -i \vec{k} \cdot \vec{r}] \bar{U}(\tau) \exp (i \vec{k} \cdot \vec{r}),
\end{aligned}
$$


where we have used $\vec{k} \cdot \vec{r}$ to denote formally the expression $K x+m \theta-1 \zeta$, and $\bar{U}(\tau)$ is the ensemble averaged propagator which satisfies the equation

$$
\left(\frac{\partial}{\partial t}-\frac{V_{D}}{r} \frac{\partial}{\partial \zeta}\right) U(t)=\frac{c}{H \zeta}\left(\frac{\partial \tilde{\phi}}{\partial \theta} \frac{\partial}{\partial r}-\frac{\partial \tilde{\phi}}{\partial \theta}\right) U(t) \text {. }
$$

Introducing the propagator $U$ is equivalent to taking into account the effect of the fluctuations on the trapped electron orbits. The problem now reduces to finding the value of $\bar{U}(\tau) \exp (\vec{i} \cdot \vec{r})$. This is achieved by operating Eq. (10) on $\exp (i \bar{k} \cdot \bar{r})$ and taking the ensemble avcragc,

$$
\begin{aligned}
\left(\frac{\partial}{\partial t}-\frac{v_{D}}{r} \frac{\partial}{\partial \zeta}\right)\langle U(t) \exp (i \vec{k} \cdot \vec{r})\rangle= & \frac{\langle c}{r B}\left(\frac{\partial \tilde{\phi}}{\partial \theta} \frac{\partial}{\partial r}-\frac{\partial \tilde{\phi}}{\partial r} \frac{\partial}{\partial \theta}\right), \\
& U(t) \exp (i k \cdot r)\rangle,
\end{aligned}
$$

where $\langle A\rangle$ denotes the ensemble average of $A$. Equation (11) has the same form as the equation for $\bar{f}$, namely

$$
\left(\frac{\partial}{\partial t}-\frac{v_{D}}{r} \frac{\partial}{\partial \zeta}\right) \bar{f}=\frac{c}{r B}\left\langle\frac{\partial \tilde{\phi}}{\partial \theta} \frac{\partial}{\partial r}-\frac{\partial \tilde{\phi}}{\partial r} \frac{\partial}{\partial \theta} f\right\rangle .
$$

Therefore, $\bar{U}(t) \exp (i \vec{k} \cdot \vec{r})$ and $\bar{f}$ satisfy the same equation. Substituting Eq. (9) into the right side of Eq. (12) and performing the ensemble average, we obtain for $\bar{f}$

$$
\left(\frac{\partial}{\partial t}-\frac{v_{D}}{r} \frac{\partial}{\partial \zeta}\right) \bar{f}=\left(\frac{1}{r} \frac{\partial}{\partial r} r D \frac{\partial}{\partial r}+\frac{1}{r} \frac{\partial}{\partial r} r G\right) \bar{f} .
$$

where

$$
\begin{aligned}
& D=\sum_{1, m}\left(\frac{m c}{r B}\right)^{2}\left|\phi_{1 m}\right|^{2}(2 a|\mu|)^{-1} \int_{-\infty}^{+\infty} \operatorname{dKR}(-K), \\
& G=\sum_{1, m}\left(\frac{m c}{r B}\right) \frac{e \omega}{T}\left|\phi_{1 m}\right|^{2}(2 a|\mu|)^{-1} \int_{-\infty}^{+\infty} d K R(-K), \\
& \left.R(K)=\int_{0}^{\infty} d \tau \exp [i \omega-\nu) \tau-i \vec{k} \cdot \vec{r}\right] \bar{U}(\tau) \exp (i \vec{k} \cdot \vec{r}) .
\end{aligned}
$$


In deriving Eq. (13), we have explicitly evaluated the ensemble average by a spatial average

$$
<>=(2 \pi)^{-2}(2 a)^{-1} \int_{-\pi}^{\pi} d \theta \quad \int_{-\pi}^{\pi} d \zeta \int_{-a}^{a} d x,
$$

where $a$ is the typical radial extent of the mode. For drift waves in general, the mode is heavity damped spatially by ion Landau damping when the distance from the rational flux surface is larger than $x_{i}$, which is defined by $k_{\theta} x_{i} / L_{S} \equiv \omega / V_{i}$, the separation between the point where parallel phase velocity of the mode is equal to $v_{i}$ and the rational surface. We will take $a \approx x_{1}$ in the following calculation. It has been shown by Weinstock ${ }^{12}$ that when the ensemble averaged quantities change slowly in space and time compared with the fluctuating quantities, the random phase average of the fluctuation is equivalent to the spatial average we introduced. Other approximations we have used in deriving Eq. (13) are in performing the $x$ integration

$$
\int_{-a}^{a} d x \exp (t K x) \approx \int_{-\infty}^{+\infty} d x \exp (i K x)=2 \pi \delta(K),
$$

because the average $K$ satisfies $K a \gg 1$.

From the discussion above, $\bar{U}(t) \exp (i \vec{k} \cdot \vec{r})$ satisfies the same equation as $\bar{f}$ :

$$
\begin{aligned}
\frac{\partial}{\partial t}-\frac{V_{D}}{r} \frac{\partial}{\partial \zeta} \bar{U}(t) \exp (i \vec{k} \cdot \vec{r})= & \left(\frac{1}{r} \frac{\partial}{\partial r} r D \frac{\partial}{\partial r}+\frac{1}{r} \frac{\partial}{\partial r} r G\right) \\
& \bar{U} \exp (i \vec{k} \cdot \vec{r}) .
\end{aligned}
$$

This equation can be solved by neglecting the slow radial derivatives, i.e., operating with $\partial / \partial r$ on the fluctuation length scale only. The solution is then 


$$
\begin{aligned}
\bar{U}(t) \exp (i \vec{k} \cdot \vec{r})=\exp (i m \theta & -i 1 \zeta+i K x-i \frac{l}{r} v_{D} t \\
& \left.+i K G t-K^{2} D t\right) .
\end{aligned}
$$

Substituting Eq. (18) into Eqs. (9) and (14) through (16), we have $R(K)$ $=i\left[\omega-\omega_{D}+K G+i\left(K^{2} D+v\right)\right]^{-1}$.

$$
\begin{aligned}
g_{l m}(K)=-\frac{\left(\omega-\omega_{\star}^{\top}\right) e \phi_{1 m}(K) / T}{\omega-\omega_{D}+K G+i\left(K^{2} D+v\right)} \bar{f}, \\
D=\sum_{1, m}\left(\frac{m c}{r B}\right)^{2}\left|\phi_{1 m}\right|^{2}\left\{\pi\left(h^{2}+G^{2}+4 D v\right) / a|\mu| h\right. \\
\left.\quad \times\left[(4 D \bar{\omega})^{2}+\left(h^{2}+G^{2}+4 D v\right)^{2}\right]^{1 / 2}\right\} \\
G=-\sum_{1, m}\left(\frac{m c}{r B}\right) \frac{e \omega}{T}\left\{\pi\left|\phi_{1 m}\right|^{2}\left(h^{2}+G^{2}+4 D v\right) / a|\mu| h\right. \\
\left.\quad \times\left[(4 D \bar{\omega})^{2}+\left(h^{2}+G^{2}+4 D v\right)^{2}\right]^{1 / 2}\right\}
\end{aligned}
$$

where

$$
\begin{aligned}
& h^{2}=\left[\left(G^{2}+4 D \nu\right)^{2}+16(D \tau)^{2}\right]^{1 / 2}, \\
& \bar{\omega}=\omega-\omega_{D}, \\
& \omega_{D}-1 V_{D} / r .
\end{aligned}
$$

Equation (19) gives the nonlinear response of trapped electrons in terms of a diffusion coefficient $D$ and a phase shift $G$. They are coupled together and related to the turbulent spectrum. The interesting point about the present result is that only the radial wave number appears explicitly in the nontinear response. The effective collision frequency due to the turbulence is $K^{2} D$ instead of $k_{\theta}^{2} D$ or $\left(k_{\theta}^{2}+K^{2}\right) D$ as previously thought. Besides the effective collision frequency, the turbulence also introduces a nonlinear phase shift to the mode frequency, as first pointed out by 
Weinstock. ${ }^{13}$ However, in our case the phase shift term can be safely neglected because $K^{2} D / K G \sim K(d \ln N / d r)^{-1} \gg 1$. When $G$ is taken to be zero, Eq. (20) can be used to solve for $D$ self-consistently. The result is

$$
D^{3 / 2}=\sum_{1, m}\left(\frac{m c}{r B}\right)^{2} \frac{\left[\pi\left|\phi_{1 m}\right|{ }^{2}\left[\nu+\left(\nu^{2}+\bar{\omega}^{2}\right)^{1 / 2}\right]^{1 / 2}\right.}{8^{1 / 2} a|\mu|\left(\nu^{2}+\bar{\omega}^{2}\right)^{1 / 2}} .
$$

From Eq. (22) we can calculate D directly if the turbulence spectrum is known. Integrating Eq. (19) over all K, we obtain

$$
\begin{aligned}
g_{1 m}= & -\left(\omega-\omega_{*}^{\top}\right) \frac{e \phi_{1 m}}{T} \bar{f}(2 \pi i \mu)^{-1 / 2} \\
& \int_{-\infty}^{+\infty} \frac{d K \exp \left[i\left(K^{2} / 2 \mu\right)+i K x\right]}{\omega-\omega_{D}+f\left(K^{2} D+v\right)},
\end{aligned}
$$

where

$$
g_{1 m}=\int_{-\infty}^{+\infty} d K g_{1 m}(K) \exp (i K x) .
$$

The $K$ integration in Eq. (23) cannot be evaluated without further approximation. We assume that the numerator is the most rapidly varying function of $K$ in the integral and evaluate it by the method of steepest descent. The result is

$$
g_{1 m}=-\frac{e \phi_{1 m}}{\cdot T} \bar{f} \frac{\left(\omega-\omega_{*}^{\top}\right) \exp \left(-i \mu x^{2} / 2\right)}{\omega-\omega_{D}+i\left[v+(x \mu)^{2} D\right]} .
$$


III. NONLINEAR DISPERSION RELATION

We observe from Eq. (24) that besides the $x$ dependence from $\phi_{1 \mathrm{~m}}$, the nonadiabatic trapped electron response $g_{1 \mathrm{~m}}$ also depends on $x$ through the turbulent collision term. In order to be self-consistent, this $x$ dependence must be treated perturbatively. After the ion response is taken into account, the final dispersion relation reduces to

$$
\begin{aligned}
1 & \left.+\left(1-\Gamma_{0}\right)-\omega_{\star} / \omega\left[\Gamma_{1}-\Gamma_{0}\right)\right] \\
& =4\left(\frac{2 L C}{\pi}\right)^{1 / 2} \int_{0}^{m} y^{2} d y \exp \left(-y^{2}\right) \int_{-\infty}^{+\infty} \frac{d x\left(\omega-\omega_{*}^{\top}\right) \exp \left(-i \mu x^{2}\right)}{\omega-\omega_{D}+i\left[\nu+(x \mu)^{2} D\right]} \\
& {\left[\int_{-\infty}^{+\infty} d x \exp \left(-i \mu x^{2}\right)\right]^{-1}, }
\end{aligned}
$$

where $\tau=T_{e} / T_{i}, y=v / V_{e}$, and $\varepsilon=r / R$ is the inverse aspect ratio. The left side of Eq. (25) is due to the adiabatic response of electrons and the lowest order ion response, while the first term on the right side is the trapped electron nonadiabatic response and the second term is the shear stabilization term. The $x$ integral in the trapped electron contribution is the result of the perturbation calculation. We aqain approximate the integral because of the difficulty of analytic evaluation. Treating the $x$ dependence in the denominator as slowly varving compared with $\exp \left(-i \mu x^{2}\right)$, we substitute a typical value for $x$ (namely, $\mu^{-1 / 2}$ ) into the denominator. The eigenmode frequency obtained by treating both the trapped electron term and the shear term as small can then be written as

$$
\omega \approx \omega_{0}+i j,
$$

where 


$$
\omega_{0}=\omega_{\star}\left[\Gamma_{0}+\eta_{i} b\left(\Gamma_{i}-\Gamma_{0}\right)\right] /\left[1+\tau\left(1-I_{0}\right)\right],
$$

and

$$
\begin{aligned}
J / \omega_{0}= & \left\{\operatorname { I m } 4 ( \frac { 2 \varepsilon } { \pi } ) ^ { 1 / 2 } \int _ { 0 } ^ { \infty } \left[y^{2} d y \exp \left(-y^{2}\right)\right.\right. \\
& \left.\left.\left(\omega-\omega_{\star}^{\top}\right)\right] /\left[\omega-\omega_{D}+1(\nu+\mu D)\right]-\left|\frac{L_{n}}{L_{S}}\right| \frac{\omega \star}{\omega_{0}^{2}}\left(D_{1} D_{2}\right)^{1 / 2}\right\} \\
& {\left[1+\tau\left(1-\Gamma_{0}\right)\right]^{-1} . }
\end{aligned}
$$

Equations (22) and (26) constitute the basic equations of our nonlinear theory. In the linear phase of the instabilities, $\left|\phi_{1 \mathrm{~m}}\right|$ is so small that $\mu D$ is much less than $\nu$. Then Eq. (26) gives us the 1 inear growth rates. As the linearly unstable modes grow, $D$ also increases until it is large enough that the growth rate of the most unstable mode is reduced to zero. A shortcoming of our theory is that the fluctuating spectrum cannot be determined within the framework of this theory. In order to relate $D$ and the fluctuation level through Eq. (22), we must assume the shape for the turbulence spectrum.

Taking $m \approx l q$, which is the condition for the relevant modes, and changing a variable to $b$, we can rewrite the summation in Eq. (22) into an integral:

$$
D^{3 / 2}=\frac{\pi}{4} r T^{2} \frac{T_{1}}{M_{i}} \int_{0}^{\infty} d b^{1 / 2}|\Phi(b)|^{2} \lambda\left(S_{2} / S_{1}\right)^{1 / 2},
$$

where

$$
\begin{aligned}
& \Phi(b)=e \phi(b) / T_{e}, \\
& \lambda^{2}=\left[\nu+\left(\nu^{2}+\bar{\omega}^{2}\right)^{1 / 2}\right] /\left(v^{2}+\bar{\omega}^{2}\right) .
\end{aligned}
$$

When $\Phi(b)$ is known we can perform the integration in Eq. (27) and obtain D. With $D$ known, we can then find the nonlinear growth rate from Eq. (26). Conversely, if we assume the instability to be stabilized by the turbulent 
collisions of the trapped electrons, we can determine $D$ from Eq. (26) such that the most unstable mode has a zero nonlinear growth rate. With the knowledge of D, we can use Eq. (27) to find the fluctuation level for an assumed shape of spectrum and then find the particle and heat fluxes due to trapped electron instability using the expressions given in Sec. IV.

IV. PARTICLE AND ENERGY FLUXES

The cross field electron particle and energy fluxes, $\Gamma_{e}$ and $Q_{e}$, can be written as

$$
\begin{aligned}
& \Gamma_{e}=\left\langle\frac{c}{B} \hat{n} \times \vec{\nabla} \phi \cdot \tilde{r N}_{e}\right\rangle, \\
& Q_{e}=\left\langle\frac{c}{B} \hat{n} \times \nabla \phi \cdot \hat{r} \int d v+\frac{m_{e} v^{2}}{2}\right\rangle .
\end{aligned}
$$

It is easy to show that onty the nonadiabatic trapped electron response contributes to Eqs. (28) and (29). We obtain from Eq. (28)

$$
\begin{gathered}
\Gamma_{e}=\left\langle\sum_{l m} \int_{-\infty}^{+\infty} d K \int_{-\infty}^{+\infty} d K^{\prime} i K_{\theta}(c / D) \phi_{-1-m^{\prime}}\left(K^{\prime}\right) d \vec{v} g_{1 m}(K)\right. \\
\left.\exp \left\lfloor i\left(K+K^{\prime}\right) x\right\rfloor\right\rangle .
\end{gathered}
$$

After carrying out the ensemble average and expressing the sum over 1 and III as an integral over $b$, we get

$$
\begin{aligned}
\Gamma_{e}=- & \frac{\pi}{2} \frac{c T_{e}}{e B} \frac{r}{2 \rho_{i}} \int_{0}^{\infty} d b\left(\frac{S_{1}}{S_{2}}\right)^{1 / 2}\left|\Phi(b)^{2}\right|_{2} \\
& \int d \vec{v}\left(\omega-\omega_{\star}^{\top}\right) D^{-1 / 2} \lambda \bar{f}
\end{aligned}
$$

and 


$$
\begin{gathered}
Q_{e}=-\frac{\pi}{2} \frac{{ }^{c T} e}{e B} \frac{r}{2 \rho_{\dagger}} \int_{0}^{\infty} d b\left(S_{2} / S_{1}\right)^{1 / 2}|\Phi(b)|^{2} \\
\int d \vec{v} \frac{m_{e} v^{2}}{2}\left(\omega-\omega_{*}^{\top}\right) D^{-1 / 2 \lambda \bar{f},}
\end{gathered}
$$

where $\lambda$ is defined following Eq. (27). In general, $D$ is a function of electron energy because $\lambda$ is a function of electron energy.

For simplicity, we ignore the energy dependence of $D$ by replacing the electron energy in $\lambda$ by its thermal energy and rewrite Eq. (27) as

$$
\begin{aligned}
D^{3} / 2= & \frac{\pi}{4} r \tau^{2}\left(T_{i} / M_{i}\right) \Phi_{0}^{2} \int_{0}^{\infty} d b b^{1 / 2} I(b) \\
& \lambda_{0}\left(S_{2} / S_{1}\right)^{1 / 2},
\end{aligned}
$$

where $|\Phi(b)|^{2} \equiv \Phi_{0}^{2} I(b)$, with $\operatorname{Max}(I)=1$ and $\lambda_{0}=\lambda\left(v=v_{e}\right)$.

Equations (30) and (31) can then be simplified to

$$
\begin{aligned}
& \Gamma_{e}=\left(\rho_{i} L_{s} / L_{n}^{2}\right) \hat{D}_{\tau} \xi_{1} \frac{c T}{e} \frac{d N}{d r}, \\
& Q_{e}=\left(\rho_{i} L_{s} / L_{n}^{2}\right) \hat{D}_{\tau} \xi_{2} \frac{c T_{e}}{e B} \frac{d N}{d r},
\end{aligned}
$$

where

$$
\begin{aligned}
& \xi_{1}=-\frac{1}{\xi_{0}} \int_{0}^{\infty} d b^{1 / 2 I}(b)\left(S_{2} / S_{1}\right)^{1 / 2} \\
& \int d \vec{v}\left(\omega-\omega_{\star}^{\top}\right) \lambda / \omega_{\star}, \\
& \xi_{2}=-\frac{1}{\xi_{0}} \int_{0}^{\infty} d b b^{1 / 2 I(b)}\left(S_{2} / S_{1}\right)^{1 / 2} \\
& \int d v \frac{m_{e} v^{2}}{2 T}\left(\omega-\omega_{\star}^{\top}\right) \hat{\lambda} / \omega_{\star}, \\
& \hat{\lambda}^{2}=\left\{\nu_{\star}+\left[\nu_{\star}^{2}+y^{b}\left(\bar{\omega} / \omega_{b}\right)^{2}\right]^{1 / 2}\right\} y^{3} /\left[\nu_{\star}^{2}+y^{b}\left(\bar{\omega} / \omega_{b}\right)^{2}\right],
\end{aligned}
$$




$$
v_{\star}=v\left(v=v_{e}\right) / \omega_{b}, \quad \omega_{b}=\varepsilon^{1 / 2} v_{e} / R q,
$$

and $\hat{D}=D\left(L_{n} / \tau \rho_{f}\right)^{2}\left(T_{i} / M_{i}\right)^{-1 / 2} / L_{S}$ is the normalized $D$.

From Eqs. (33) and (34), we observe that both $\Gamma_{e}$ and $Q_{e}$ are proporttonal to $D$. This is consistent with our previous notion that $D$ is the nonlinear spatial diffusion. The collision frequency dependence of $\Gamma_{e}$ (or $Q_{e}$ ) is contained in $\hat{D} \xi_{1}$ (or $\hat{D} \xi_{2}$ ). From the numerical calculation in Sec. $V$, it is seen that $\hat{D}$ is roughly constant in the low collision frequency limit. In the same limit, $\xi_{1}$ and $\xi_{2}$ are also approaching constant values. Thus, the anomalous transport assoclated with the dissipative trapped electron mode persists even in collisionless regimes.

\section{NUMERICAL EXAMPLE AND DISCUSSION}

The theory described in Secs. 2-4 is illustrated by the following example in which we numerically evaluate the nonlinear trapped electron growth rate in Eq. (26) as $D$ increases from zero.

In Fig. 1, we have plotted the nonlinear growth rate due to trapped electrons for different values of $\hat{D}$ against the poloidal mode number parameter $b=\left(k_{\theta} \rho_{j}\right)^{2}$ in a solid line. The shear contribution to the damping rate is plotted separately in a dashed line. The total growth rate is therefore the difference between the solid line and the dashed 1ine. The relevant parameters used in this example are: $\eta_{e}=\eta_{j}=1$, $\varepsilon=0.15, L_{n} / R=0.25, q=2, \tau=1, L_{n} / L_{S}=0.05$, and $\nu_{\star}=0.01$. A11 the growth rates shown in Fig. 1 are normalized by $\left(T_{i} / M_{i}\right)^{1 / 2} / L_{n}$. 
When $\hat{D}$ is zero or much smaller than $v_{*}$, the trapped electron contribution to the growth rate is the same as that given by linear theory. There is a peak in the growth rate around $b \simeq 0.2$. As $\hat{D}$ increases from zero, the peak growth rate due to trapped electrons becomes smaller and the curve is broadened at the same time. The location of the peak in this particular case happens to remain about the same. This indicates that as the turbulence grows, the most unstable mode reduces its growth rate by turbulent collision damping. However, modes at larger $b$ are destabilized. We conclude that the free energy available for the instability is spread out in $k_{\theta}$ space by the broadening effect of turbulent collisions. When $\hat{D}$ is larger than 0.2 , the trapped electron growth rate is smaller than the shear damping rate for all $k_{\theta}$, and the instability is completely stabilized.

Critics of the renormalized turbulence theory will argue at this point that as $\hat{D}$ increases, more and more modes are stabilized by shear and their fluctuation amplitudes decay in time until finally only the most unstable mode is left. Such a final state is not consistent with the turbulent assumption of the theory. Several answers to this question are possible. The first possibility is that due to the broadening effect of the turbulent collisions, the modes near the most unstable mode have very slow damping rates, so that their fluctuation amplitudes change only a little during the lifetime of the plasma. This effect will provide enough stochasticity to the system. Another possibility is that initially there are many unstable modes in the system, so that it can be correctly described by turbulence theory until very close to marginal stability where only a few unstable modes are left. Then some form of mode coupling 
scheme must be developed to determine the final saturated state. In that case, the renormalized turbulence theory should still give a fairly good qualitative description of the saturated state. Finally, the recent clump theory of Dupree ${ }^{14}$ extends the renormalized turbulence theory to include two-particle correlations as a source term in the dispersion relation in such a way that the single mode final state can be avoided. However, refinement of our theory to include clumps is beyond the scope of this paper.

We can obtain sets of curves similar to Fig. 1 for different values of $\nu_{*}$. As a result, the value of $\hat{b}$ needed to stabilize the instability for a given shear strength, $\hat{D}_{c}$, can be obtained as a function of $v_{\star}$. This function is plotted in Fig. 2 for a few values of the shear parameter $L_{n} / L_{S}$. We observe from this figure that $\hat{D}_{c}$ is fairly constant over low values of $v_{*}$ and drops to zero rapidly when $v_{*}$ is large enough that the Coulomb collision alone is able to stabilize the instability.

Having $\ddot{D}_{c}$ known, we can find $\theta_{0}$ from Eq. (32). The equation rclating $\hat{D}_{c}$ and $\Phi_{0}$ can be written as

$$
\ddot{\Phi}_{u}^{2}=\frac{4}{\pi}\left(\frac{\rho_{q}}{L_{n}}\right)^{3} \tau^{5 / 4}\left(\varepsilon \frac{M_{q}}{m_{e}}\right)^{1 / 4} \frac{L_{g}}{r}\left(\frac{L_{g}}{R q}\right)^{1 / 2} \frac{\hat{D}_{u}^{3 / 2}}{\xi_{0}} .
$$

In Eq. (35), the $\nu_{\star}$ dependence of $\Phi_{0}$ is contained in $\hat{D}_{c} 3 / 2 / \xi_{0}$. for an assumed turbulence spectrum $I(b)$ peaked around the most unstable mode, $I(b)=\exp \left[-\left(b-b_{0}\right)^{2}\right]$, where $b_{0}$ is the location of the most unstable mode; this quantity is plotted in Fig. 3 for the same parameters used in Fig. 1. It is seen that $\hat{D}^{3} /: 2 / \xi_{j}$, or $\Phi_{0}$, starts from zero near $v_{\star} \sim 1$, increases quickly to its peak value at a slightly lower collisionality, and then 
drops back to an asymptotic value at low $\nu_{\star}$. For the parameters used in Fig. $I$ and further assuming $\rho_{i}=0.2 \mathrm{~cm}$ and $L_{n}=20 \mathrm{~cm}$, we get from Eq. (35) $\Phi_{0}^{2} \sim(0.019)^{2} \hat{D}_{c}^{3 / 2 / \xi_{0}}$. When $\hat{D}_{c} 3 / 2 / \xi \sim 0.1$, it gives $\Phi_{0} \sim 0.6 \%$, which is not far from the values observed experimentally.

The particle and energy fluxes can be calculated from Eqs. (33) and (34). The $\nu_{*}$ dependences of $F_{e}$ and $Q_{e}, \hat{I}$ and $\hat{Q}$, namely the factors $\hat{D}_{c} \xi_{1}$ and $\hat{D}_{c} \xi_{2}$, are plotted in Fig. 4. For the parameters used, Eqs. (33) and (34) also give diffusion coefficients consistent with experimental values. For comparison, the neoclassical $\hat{\Gamma}$ is also plotted in Fig. 4.

VI. CONCLUSION

We have shown that turbulence-induced collisions acting on trapped electrons are very significant in the nonlinear saturation of the dissipative trapped electron instability, particularly when the plasma is deep in the banana regime. When the shape of the filuctuation spectrum is assumed to be similar to those observed in experiments, i.e., peaked around $k_{\theta} \rho_{i} \simeq 1$, the fluctuation level and diffusion coefficients obtained from the theory are consistent with the experimental values.

\section{ACKNOWLEDGMENTS}

We would like to thank T. H. Dupree, D. J. Tetreault, and P. J. Catto for useful discussions and J. Whitson for programming assistance.

This research was sponsored by the Energy Research and Development Administration under Contract No. W-7405-eng-26 and Contract No. E(40-1)5260. 
APPENDIX

Weak versus Strong Plasma Turbulence

For completeness, in this appendix we briefly comment on the concepts of weak and strong turbulence, i.e., on the relationships between "standard" weak plasma turbulence and Navier-Stokes fluid turbulence theories. (An illuminating review can be found in a recent paper by Montgomery.15) It is to be noted that we are not concerned here with the potential merits of the large subset of papers dealing with varlous aspects of renormalization in a weak plasma turbutence.

The equations of motion of either a plasma or a fluid can be written in the matrix operator form

$$
\left(\frac{\partial}{\partial t}+i L\right) f=N f
$$

where $L$ is a linear operator on $f$ and $N$ is a nonlinear (bilinear) operator on $f$. The column matrix $f$ stands for either particle distribution functions (plasmas); velocity, pressure, and magnetic fluids (magnetohydrodynamics); or velocity field (incompressible Navier-Stokes fluids). The relevant boundary and/or initial conditions are assumed to be given.

In linear theory, one assumes

$$
\left\|L f(0)_{\|} \gg\right\| N f(0)_{\|}
$$

introduces, for example, Fourier transforms, and then proceeds to solve

$$
\left(\frac{\partial}{\partial t}+i L\right) f^{(0)}=0=(-\omega I+L) f^{(0)}
$$

as an eigenvalue problem, under the given conditions. I is the identity operator. After finding the spectrum of the operator $(-\omega I+L)$, one finds 
the required solution $f^{(0)}$ by summing over the point spectrum $\left\{\omega_{1}^{(0)}\right.$, $\left.\omega_{2}^{(0)}, \ldots\right\}$ and integrating over the continuous spectrum contributions. This immediately leads to the concept of a "linear dispersion relation" for $j^{\text {th }}$ with $\omega=\omega_{j}^{(0)}(k)$. The real part of $\omega_{j}^{(0)}(k)$ is called the "frequency" of the $j^{\text {th }}$ wave and it is classified as "stable" or "unstable" depending on the sign of the imaginary part of $\omega_{j}^{(0)}(k)$.

In Navier-Stokes turbulence, the linear operator $i L=k^{2} v I$, where $v$ is the kinematic viscosity. Now in the region of interest, $v \ll 1$, so that $\|L f\|_{N \cdot S} \ll\|N f\|_{N \cdot S}$. That is, there is no meaningful linear theory to give rise to "dispersion relations," "waves," "instabilities," etc. It should be noted that the $\underset{\sim}{E} \times \underset{\sim}{B} \mathrm{drift}$ approximation to the Vlasov equation is equivalent to the two-dimensional, inviscid, incompressible NavierStokes equation, and so the above remarks apply to purely two-dimensional plasma drift turbulence as well. It is thus of interest to note that just as the random phase approximation leads to unphysical results ${ }^{16}$ in fluid turbulence, so also does this approximation applied to purely two-dimensional plasma drift turbulence (see Cook's comments' ${ }^{17}$ on the TaylorThompson theory $\left.{ }^{18}\right)$. Thus from the outset, Navier-Stokes turbulence is a nonlinear problem. The major breakthrough was achieved by Kraichnan, i9 who obtained a closed set of integro-differential equations (the "direct interaction approximation"). This closed set of equations is of such formidable complexity that no significant analytic consequences can be derived from them, and this is for the simplest geometry possible (a large cubic box, assuming periodicity). The importance of Kraichnan's equations is that their subsequent numerical solution ${ }^{20}$ gave remarkable agreement with experiment. (Nevertheless, some unsatisfactory features were found and 
this has led to more complicated generalizations; see, for example, a review by Krafchnan. ${ }^{21}$ ) It is of interest to note that this approximation has been applied by Montgomery 15 to a one-dimensional electron plasma with an immobtle ion background with periodic boundary conditions. The resulting closed set of equations appears at the present time to be numerically unmanageable.

Now in plasma physics, the linear problem in Eq. (A3) docs yicld nontrivial and significant results. In particular, it yields a "linear dispersion relation," "particle-wave interaction," etc. The question then arises as to the outcome of a particular linear instabtlity (derived from the linear dispersion relation). The weak plasma turbulence approach is to assume that to include the relevant nonlinear effects one needs only to modify the linear theory appropriately. Quasi-linear theory treats the nonlinear term $N f$ as a perturbation on the linear term iLf. However, this theory leads to time secularities. Dupree then introduced the concept of renormalization by including the relevant part, say $N_{1} f$, of the nonlinear term to modify $1 L f$ and then treating the nonlinearity $\left(N-N_{1}\right) f$ perturbatively: The advantages of this theory are that one can rely fairly heavily on modified linear instability intuition via a "nonlinear dispersion relation," "broadened particle-wave resonance," etc. One of the chief drawbacks is that the resulting Dupree equations are not a closed set. For example, we had to:assume the structure of the turbulent spectrum [see Eq. (27)] to obtain the closure needed to investigate nonlinear saturation of the mode. The standard mode-mode coupling theories also belong to the weak turbulence category because they suitably modify the linear problem by perturbation techniques. The strong plasma 
turbulence approach assumes that the resulting turbulence from the linear instability is such that linear theory concepts are no longer applicable (cf. Navier-Stokes turbulence). Little progress has been made in this area (however, see, for example, Ichtmaru22).

It is hoped that for low $\beta$ plasmas, the resulting turbulence can be treated in the Dupree format, while for high $\beta$ plasmas, strong (NavierStokes type) turbulence can be expected to ensue.

\section{REFERENCES}

${ }^{1}$ E. Mazzucato, Phys. Rev. Lett. 36, 792 (1976).

${ }^{2}$ TFR Group, Plasma Physics and Controlled Nuclear Fusion Research 1976,: (IAEA, Vienna, 1977), Vol. I, p. 35.

3W. M. Manheimer, E. Ott, K. R. Chu, J. P. Boris, and J. D. Callen, Nucl. Fusion 16, 203 (1976).

${ }^{4}$ C. Z. Cheng and H. Okuda, Phys. Rev. Lett. 38, 708 (1977).

${ }^{5}$ T. H. Dupree, Phys. Fluids 11, 2680 (1968).

${ }^{6} \mathrm{H}$. W. Hendel, T. K. Chu, and P. A. Politzer, Phys. Fluids 11,2426 (1968).

7B. B. Kadomtsev and 0. P. Pogutse, Dok1. Akad. Nauk SSSR [Sov. Phys.Doklady] 14, 470 (1969).

8J. C. Adam, W. C. Tang, and P. H. Rutherford, Phys. Fluids $\underline{19}, 561$ (1976).

9K. T. Tsang, J. D. Callen, and P. J. Catto, Collisional Effects of Trapped Electron Instabilities, ORNL/TM-5236, Oak Ridge National Laboratory, Oak Ridge, Tennessee (1977); submitted to Phys. Fluids. 
${ }^{10} \mathrm{~B}$. B. Kadomtsev and 0. P. Pogutse, Reviews of Plasma Physics (Consultants Bureau, New York, 1970), Vol. 5, p. 249.

11B. V. Wadde11, Nucl. Fusion 15, 803 (1975).

12J. Weinstock, J. Geophys. Res. 81, 633 (1976).

13J. Weinstock, Phys. Fluids 15, 454 (1972).

${ }^{14}$ T. H. Dupree, Phys. Fluids $\underline{15}, 334$ (1972).

${ }^{15} \mathrm{D}$. Montgomery, Univ. of Iowa Report $7 G=33$ (to be published).

16y. Ogura, J. Fluid Mech. 16, 33 (1963).

1\%I. Cook, J. Plasma Phys. 12, 501 (1974).

18J. B. Taylor and W. B. Thompson, Phys. Fluids 16, 111 (1973).

19 R. H. Kraichnan, Phys. Rev. 113, 1181 (1958); J. Fluid Mech. 5, 497

(1959); J. Math. Phys. 2, 124 (1961).

${ }^{20}$ R. H. Kraichnan, Phys. Fluids 7,1030 (1964).

21 R. H. Kraichnan, Adv. Math. 16, 305 (1975).

22S. Ichimaru, Phys. Fluids 13, 1560 (1970). 


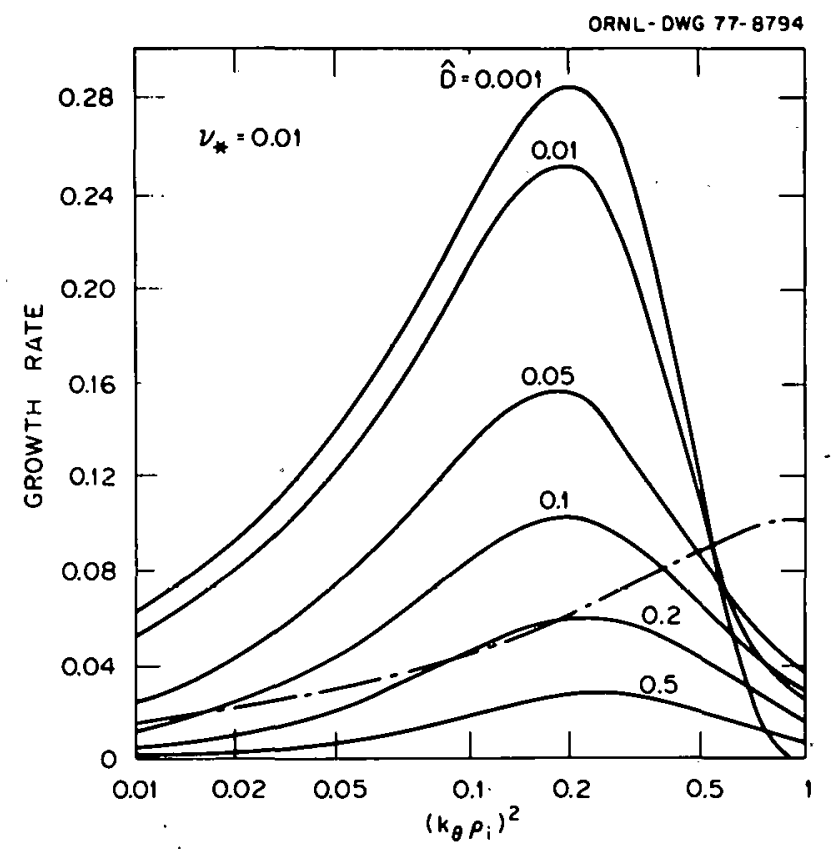

Fig. 1. Nonlinear trapped electron growth rate for $\hat{D}=0.001,0.01,0.05,0.1,0.2$, and 0.5 . The relevant parameters are $n_{e}=\eta_{i}=1, \varepsilon=0.15, L_{n} / R=0.25, q=2$, $\tau=1, L_{n} / L_{S}=0.05$, and $\nu_{*}=0.01$. The dashed line is the shear damping rate. 


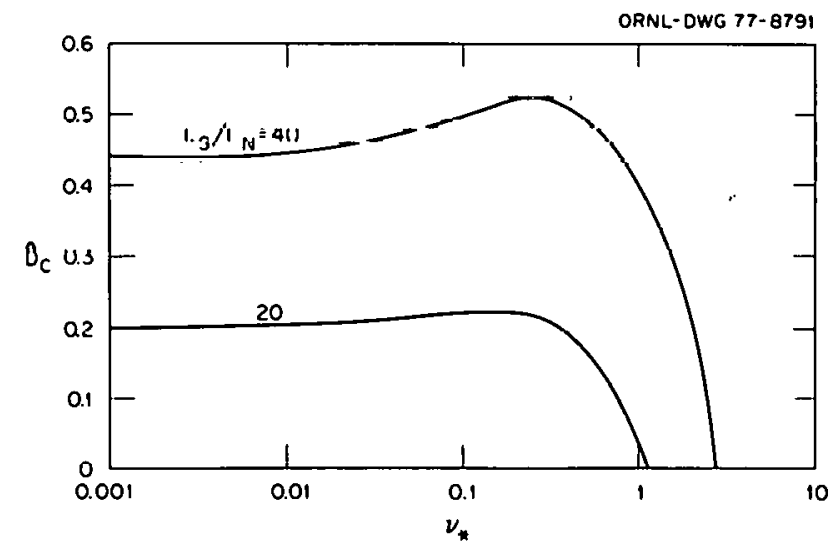

Fig. 2. $\hat{\mathbf{D}}$ needed to stabilize the instability for a given shear strength as a function of $\nu_{\star}$. 


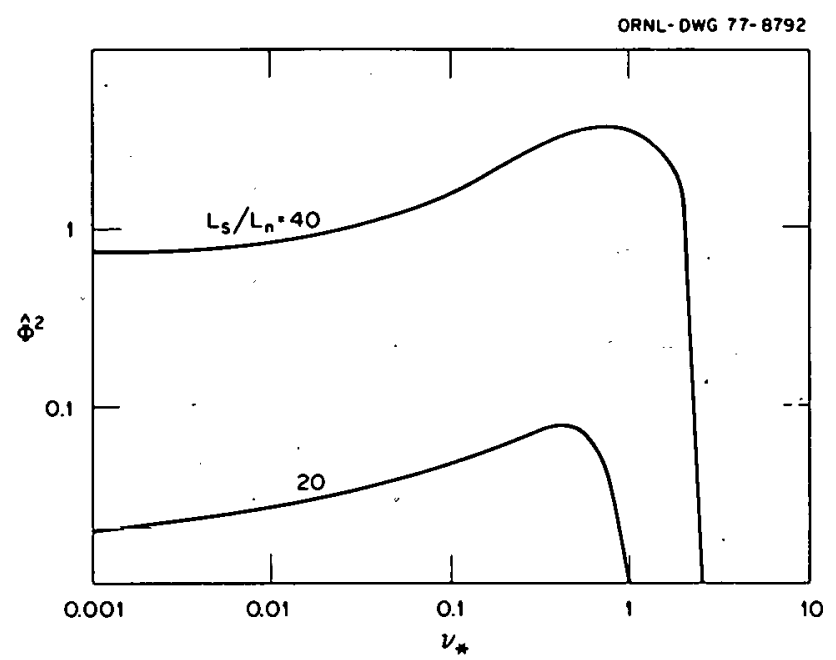

Fig. 3. Normalized fluctuation level $\hat{\Phi}_{0}$ as a function of $\nu_{\star}$ for a given shear. 


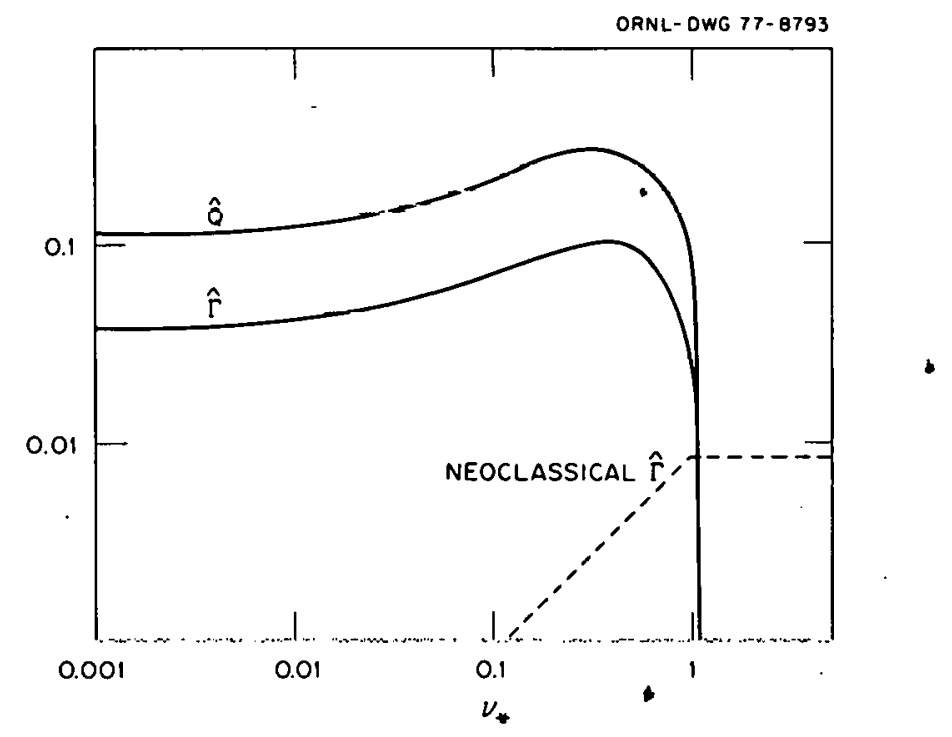

Fig. 4. Normalized particle and energy fluxes, $\hat{\Gamma}$ and $\hat{Q}$, as functions of $\nu_{\star}$ for $L_{S} / L_{n}=20$. For comparison, the neoclassical $\hat{\Gamma}$ for the same case is also plotted. 
ORNL/TM-5942

\section{INTERNAL DISTRIBUTION}

1. J. D. Callen

2. J. F. Clarke

3. R. A. Dory

4. G. G. Kelley

5. 0. B. Morgan

6. M. W. Rosenthal

7-31. K. T. Tsang

32-34. Laboratory Records Department

35. Laboratory Records, ORNL - RC

36. $Y-12$ Document Reference Section

37-38. Central Research Library

39. Fusion Energy Division Library

40. Fusion Energy Division Reports Office

41. ORNL Patent Office

EXTERNAL DISTRIBUTION

42. Plasma Physics Library, Plasma Physics Laboratory, Princeton Univ., Forrestal Campus, P.0. Box 451, Princeton, NJ 08540

43. Controlled Thermonuclear Research Library, Lawrence Livermore Laboratory, P.0. Box 808, Livermore, CA 94550

44. Q Division Library, Los Alamos Scientific Laboratory, P.0. Box 1663, Los Alamos, NM 87544

45. Controlled Thermonuclear Research Library, c/o Weston M. Stacey, Jr., Argonne National Laboratory, 9700 S. Cass Ave., Argonne, IL 60439

46. CTR Computer Center, c/o Dr. John Killeen, Lawrence Livermore Laboratory, P.0. Box 808, Livermore, CA 94550

47. Librarian, Culham Laboratory, U.K. Atomic Energy Authority, Abingdon, 0xon, 0X14 3DB, United Kingdom

48. Ruth Lengye, Bibliothek, Max-Planck Institut für Plasmaphysik, 8046 Garching bei München, Federal Republic of Germany

49. Library, Centre de Recherches en Physique des Plasmas, 21 Avenue des Bains, 1007, Lausanne, Switzerland

50. A. M. Dupas, Documentation S.I.G.N., Départment de la Physique du Plasma et de la Fusion Controlee, Association EURATOM-CEA sur la Fusion, Centre d'Etudes Nucléaires, BP 85 Centre Du TRI 38041 Grenoble Cedex (France)

51. Bibliotheque, Service du Confinement des Plasmas, C.E.A., B.P. No. 6, 92, Fontenay-aux-Roses (Seine) France

52. Library, International Centre for Theoretical Physics, Trieste, Italy

53. Library, Laboratorio Gas Ionizzati, Frascati, Italy

54. V. E. Ivanov, Physical-Technical Institute of the Ukranian Academy of Sciences, Sukhumi, U.S.S.R.

55. L. M. Kovrizhnikh, Lebedev Institute of Physics, Academy of Sciences of the U.S.S.R., Leninsky Prospect 53, Moscow, U.S.S.R.

56. Prof. Dshumber G. Lominadze, Academy of Sciences of the Georgian SSR, 8 Dzerzhinski St., 38004, Tbilisi, USSR

57. Library, Inst. for Plasma Physics, Nagoya Univ., Nagoya, Japan 464 
58. Library, FOM-Institut voor Plasma-Fysica, Rijnhuizen, Jutphaas, Netherlands

59. Plasma Physics Group, Department of Engineering Physics, Australian National University, P.0. Box 4, Canberra A.C.T. 2600, Australia

60. Thermonuclear Library, Japan Atomic Energy Research Institute, Tokai, Naka, Ibaraki, Japan

61. Dr. D. G. McAlees, Exxon Nuclear Co., Inc., Research \& Technology Laser Enrichment Department, 2955 George Washington Way, Richland, WA 99352

62. CTR Reading Room, c/o Prof. D. W. Kerst, Dept. of Physics, Sterling Hall, Univ. of Wisconsin, Madison, WI 53706

63. CTR Reading Room, c/o Prof. I. B. Bernstein, Yale Univ., 200 Mason Laboratory, Dept. of Engineering \& Applied Science, New Haven, CT 06510

64. Center for Plasma Physics and Thermonuclear Research, c/u D. W. Russ, Physics Dept., Univ. of Texas, Aust1n, TX $7871 \%$

65. CTR Reading Room, c/o Prof. B. D. Fried, Physics Dept., Univ. of California, Los Angeles, CA 90024

66. CTR Reading Room, c/o Prof. David C. Montgomery, Physics \& Astronomy Dept., Univ. of Iowa, Iowa City, IA 52240

67. Magneto-Fluid-Dynamics Library, c/0 Dr. Harold Grad, Courant Inst. of Math. Sci., New York Univ., 251 Mercer St., New York, NY 10012

68. CTR Reading Room, c/o Prof. Allan N. Kaufman, Physics Dept., Univ. of California, Berkeley, CA 94720

69. Dr. David A. Dingee, Fusion Programs, Battelle-Northwest, Battelle Boulevard, Richland, WA 99352

70. CTR Reading Room, c/o Prof. C. S. Liu, Dept. of Physics and Astronomy, Univ. of Maryland, College Park, MD 20742

71. CTR Reading Room, c/o Prof. T. Kammash, 103 Research Admin. B1dg., N. Campus, Univ. of Michigan, Ann Arbor, MI 48105

72. CTR Reading Room, c/o Dr. Ravi N. Sudan, Phillips Hall, Cornell Univ., Ithaca, NY 14850

73. Prof. Marshali N. Rosentluth, Institute for Advanced Study, Princeton, NJ 08540

74. CTR Reading Room, c/o Prof. R. Gross, Plasma Research Lab., Columbia Univ., New York, NY 10027

75. CTR Reading Room, c/o Prof. Roy Gould, Calffurnta Inst. of Tech., M.S. 116-81, Pasadena, CA 91125

76. Dr. Nicholas A. Krall, Science Applications, Inc., P.0. Box 2354, 1200 Prospect St., La Jolla, CA 92037

77. CTR Reading Room, c/o Dr. Jay P. Boris, Plasma Physics. Nava? Research Laboratory, Washington, DC 20390

78. Professor A. Simon, Dept. of Mechantcal \& Aerospace Sciences, University of Rochester, Rochester, NY 14627

79. CTR Library, c/o Dr. Alan F. Haught, United Technologies Research Labs, East Hartford, CT 06108

80. Dr. H. K. Forsen, Exxon Nuclear Co., Inc. 777-106th Avenue, NE, C-000777, Bellevue, WA 98009

81. Dr. George Vahala, Physics Dept., College of William \& Mary, Will iamsburg, VA 23185

82. Dr. Robert E. Price, Division of Magnetic Fusion Energy, G-234, Energy Research and Development Administration, Washington, D.C. 20545 
83. Dr. R. C. Davidson, Division of Magnetic Fusion Energy, G-234, Energy Research and Development Administration, Washington, D.C. 20545

84. Dr. Oscar P. Manley, Division of Magnetic Fusion Energy, G-234, Energy Research and Development Administration, Washington, D.C. 20545

85. Mr. E. E. Kintner, Division of Magnetic Fusion Energy, G-234, Energy Research and Development Administration, Washington, D.C. 20545

86. Dr. L. D. Pearlstein, L-388, Lawrence Livermore Laboratory, P.0. Box 808, Livermore, CA 94550

87. Dr. J. P. Friedberg, Los Alamos Scientific Laboratory, Los Alamos, NM 87544

88. Dr. David J. Rose, Dept. of Nuclear Engineering, MIT, Cambridge, MA 02139

89. Dr. Gareth E. Guest, General Atomic Co., P.0. Box 81608, San Diego, CA 92138

90. Dr. Claude Mercier, Service du Theorie des Plasmas, Centre d'Etudes Nucleaires, Fontenay-aux-Roses (Seine) France

91. Dr. J. B. Taylor, Culham Laboratory, UKAEA, Abingdon, Oxon, 0X14 3DB, United Kingdom

92. Dr. D. Pfirsch, Institute for Plasma Physics, 8046 Garching bei München, Federal Republic of Germany

93. Dr. V. D. Shafranov, I. V. Kurchatov Inst. of Atomic Energy, 46 Ulitsa Kurchatova, P.0. Box 3402, Moscow, U.S.S.R.

94. Dr. A. Rogister, Institute for Plasma Physics, KFA, Postfach 1913, D-5170, Jülich 1, Federal Republic of Germany

95. Dr. J. G. Cordey, Culham Laboratory, UKAEA, Abingdon, Oxon, 0X14 3DB, United Kingdom

96. Dr. David Ba]dwin, L-388, Lawrence Livermore Lab., P.0. Box 808, Livermore, CA 94550

97. CTR Reading Room, c/o Prof. Bruno Coppi, Dept. of Physics, MIT, Cambridge, MA 02138

98. Dr. Harold P. Furth, Princeton Plasma Physics Lab., Princeton Univ., P.0. Box 451, Princeton, NJ 08540

99. Dr. Paul H. Rutherford, Princeton Plasma Physics Lab., Princeton Univ., P.0. Box 451, Princeton, NJ 08540

100. Dr. Herbert $H$. Woodson, Dept. of Electrical Engineering, Univ. of Texas, Austin, TX.78712

101. Research \& Technical Support Div., Oak Ridge Operations, Energy Research and Development Administration, P.0. Box E, Oak Ridge, TN 37830

102-128. Tech. Information Center, Oak Ridge Operations, Energy Research and Development Administration, P.0. Box 62, Oak Ridge, TN 37830 